\title{
Endoscopic Ultrasound-Guided Drainage of a Pancreatic Pseudocyst after a Bicycle Trauma
}

\section{Introduction}

Blunt pancreatic injuries are rare as they only comprise $1-5 \%$ of abdominal trauma, and half of the cases are seen in combination with multiple injuries. More than $60 \%$ of pancreatic injuries are located in the body and tail of the gland (Krige JE et al. Pancreatology. 2017; 17(4): 592-598).

Pancreatic trauma often entails severe lesions with a high morbidity and mortality if treatment is delayed or inadequate (Mohseni $S$ et al. Injury. 2018; 49(1): 27-32). Treatment is controversial and depends on whether the main pancreatic duct has been injured. Grade I and II trauma is usually managed conservatively, while grade III to V trauma is generally managed operatively either with drainage or resection of major parts of the gland (Ho VP et al. J Trauma Acute Care Surg. 2017; 82(1): 185-199). However, an increasing number of studies suggest that non-operative management with drainage alone rather than resection may provide acceptable outcomes (Menahem B etal. Hepato Biliary Surg Nutr. 2016; 5(6): 470-477).

We present an acute case with a grade III lesion of the pancreatic neck in an adult treated with surgical drainage and subsequent drainage of a pseudocyst with a lumen-apposing metal stent (LAMS) with conservation of the gland.

\section{Case Presentation}

A 27-year-old healthy female suffered a grade III lesion of her pancreas after she fell from a bicycle and landed on the handlebar. At a local hospital a pancreatic contusion was found on a trauma CT scan, and the patient was referred to a level 1 trauma center with specialized HPB function. A repeat CT scan revealed complete rupture of the pancreatic neck with a retroperitoneal hematoma without signs of other abdominal injuries ( Fig. 1). An MRCP confirmed rupture of the main duct with a diastasis measuring $2 \mathrm{~cm}$. A conservative approach was chosen, and the patient was treated with a nasogastric tube with continuous suction, intravenous proton pump inhibitor (pantoprazole $40 \mathrm{mg}$ b.i.d.), subcutaneous octreotide 100 microgram t.i.d., intravenous cefuroxime $1500 \mathrm{mg}$ t.i.d., metronidazole $1500 \mathrm{mg}$ q.d. and parenteral nutrition. On the third day of admission an endoscopic retrograde cholangiopancreatography (ERCP) with papillotomy of the pancreatic duct was performed to ease the flow to the duodenum and diminish the leakage from the severed duct. Due to the considerable diastasis of the duct ends and the large hematoma with displacement of the fractured parts, an attempt to insert a bridge prosthesis over the contused area was not attempted. On the fourth day the patient's condition deteriorated with increasing abdominal pain, inflammatory parameters and free intraperitoneal fluid seen on ultrasonography. A laparotomy was performed with removal of $2000 \mathrm{ml}$ ascites, but the surgeon refrained from resection of the distal part of the gland due to a large retroperitoneal hematoma. Instead 2 external 18 Fr tubes were placed along the superior and inferior pancreatic border, respectively, and the abdomen was closed. The patient's general condition quickly improved with no need for pain killers, the systemic inflammatory response decreased, she started eating regular food and one abdominal tube was discontinued because of the decreasing amount of fluid. In the remaining tube the level of liquid was stable around $200 \mathrm{ml} /$ day with amylase of $10000 \mathrm{U} / \mathrm{l}$. The patient was discharged on day 16 and followed up once a week at the outpatient clinic with intermittent retraction of the drain until a fistula to the skin had formed and the drain was

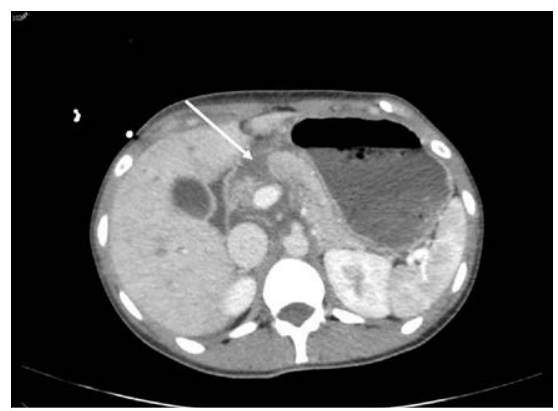

- Fig. 1 The CT scan shows trauma to the neck of the pancreas with a $2 \mathrm{~cm}$ diastasis between the head and body with retroperitoneal extravasation. removed 8 weeks later. Two weeks after removal of the drain, the discharge had ceased from the fistula and the patient complained of increasing discomfort and abdominal pain. A CT scan revealed a pseudocyst of $4.6 \times 3.1 \times 2.6 \mathrm{~cm}$ and normal contrast enhancement in both halves of the gland. An MRCP showed the severed pancreatic duct with a diameter of $6 \mathrm{~mm}$ and a large walledof fluid collection protruding in to the posterior wall af the stomach ( $\triangleright$ Fig. 2). Endoscopic ultrasonography was performed and a $10 \times 10 \mathrm{~mm}$ HOT AXIOS ${ }^{\text {TM }}$ stent (Boston scientific, Marlborough, MA) was inserted between the stomach and the cyst ( $>$ Fig. 3 and -4$)$. A therapeutic Pentax echoendoscope (EG-3870UTK; Pentax, Tokyo, Japan) and Hitachi ultrasound workstation (EUB 7500, HI Vison Preirus; Hitachi Medical Corp., Tokyo, Japan) were used. The collection was punctured under EUS guidance

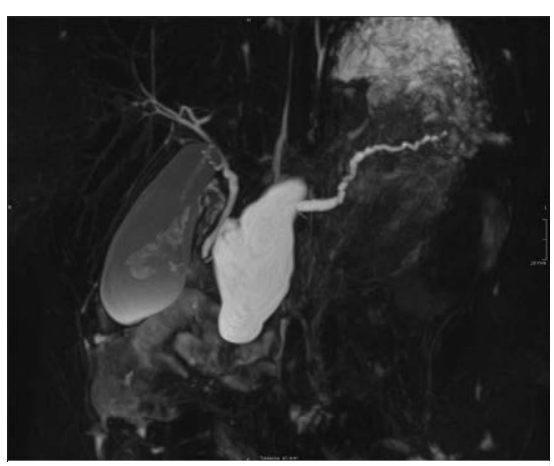

-Fig. 2 Magnetic resonance cholangiopancreatography (MRCP) showing disruption of the main pancreatic duct with formation of a large walled-of fluid collection (pseudocyst) adjacent to the stomach.

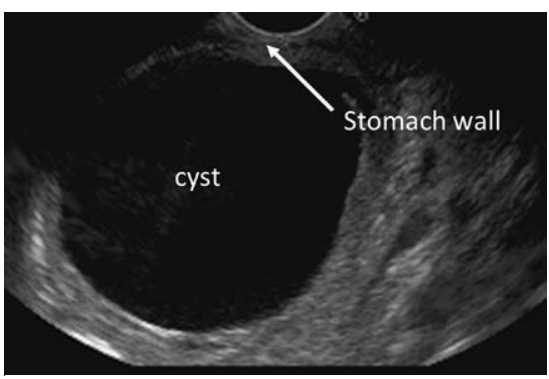

- Fig. 3 Ultrasound image of a pancreatic pseucocyst and the stomach wall. 
using the electrocautery wire at the tip of the Hot AXIOS stent. Once the device was satisfactorily positioned within the cyst, the distal flange of the stent was deployed under EUS guidance. The device was then pulled back until the distal flange deformed against the cavity wall. The proximal flange was then deployed on the luminal side under direct endoscopic control. The same evening the patient could eat normally, the abdominal pain had ceased, and she was discharged the following day. Five weeks later a CT scan revealed a collapsed cyst and nine weeks from insertion the stent was removed by regular gastroscopy. Two weeks after removal of the stent, a CT scan showed no recurrence of the cyst, the pancreatic duct still measured $6 \mathrm{~mm}$ and both halves of the pancreas had blood supply. The patient was doing well without signs of malabsorption or diabetes and the follow-ups were terminated but with open contact to our department.

\section{Discussion}

Although treatment of pancreatic pseudocysts with transgastric drainage is a wellknown procedure, definitive treatment of complications of pancreatic fracture with LAMS is not a standard procedure.

The acute treatment of pancreatic trau$\mathrm{ma}$ is damage control based on radiologic evaluation preferably from MRCP to assess the patency of the duct. The conservation of pancreatic tissue is desirable but often difficult to meet in grade III-V trauma and resection of the severed part of the gland may lead to exocrine and occasionally also to endocrine insufficiency.
There is, however, an increasing trend of nonoperative management of grade III lesions with improved survival likely reflecting an improvement in critical care, nutrition and infection management. In the acute phase it is important to drain intraperitoneal and peri-glandular pancreatic juice. This can be managed surgically or under ultrasound guidance.

Apart from acute complications such as bleeding, peritonitis, pancreatitis and systemic complications with septicemia, coagulopathy and ARDS, the usual outcome after conservatively treated pancreatic fracture would be either a persistent fistula or a pancreatic pseudocyst. In the first case of a fistula, jejunostomy is the usual treatment, while a large or expanding pseudocyst needs permanent drainage. This is usually performed via a cysto-gastrostomy, either by surgery or by stenting.

The endoscopic approach has the same efficacy as the open procedure. Endoscopically ultrasound-guided insertion of $\mathrm{Fr} 7$ or 10 pig tail plastic stents has been the standard treatment but with recurrence of the cyst in almost 20 percent of cases due to the narrow lumen of the stent (Singhal S et al. Clin Endosc. 2013; 46: 506-514). Self-expanding covered metal stents with an increased lumen have a higher patency but also a risk of migration into the cystic or gastric lumen. LAMS is a novel device consisting of a combined lumen-apposing bi-flanged metal stent and an electrocautery-enhanced delivery system. This enables a single device to be used instead of multiple devices and multiple steps. The advantage of the LAMS with its dumbbell configuration is lumen-to-lumen anchorage between the cystic and gas- tric wall, which avoids migration of the stent and provides a wider diameter than the plastic stents that facilitates better drainage in the presence of debris (Patil R et al. Ann Gastroenterol. 2016; 29: 168-173).

Complications of LAMS are late bleeding, possibly resulting from friction between the stent and the wall of the cyst and the stomach, and overgrowth of the stent with gastric mucosa, "burial stent syndrome". Until now these complications have only been casuistically reported (Bang et al. Gut. 2017; 66: 2054-2056).

We decided to choose a conservative approach in the treatment of the pancreatic injury, but an operation was necessary to ensure proper drainage of pancreatic fluid and formation of a controlled external fistula. We refrained from resection of the distal part of the gland due to a large retroperitoneal hematoma with the risk of profuse bleeding. Even if a resection had been performed, this would probably have resulted in a pancreatic fistula because permanent closure of the severed pancreatic duct would be difficult in the contused gland. By choosing this semi-conservative approach, it was possible to minimize the surgical procedure and postoperative complications as well as to preserve pancreatic tissue.

Depending on the situation and the presence of concomitant injuries, the initial treatment of grade III pancreatic lesions may be managed by drainage, and in the case of subsequent formation of a pseudocyst, a minimally invasive procedure with endoscopic LAMS insertion may be an alternative to other procedures.
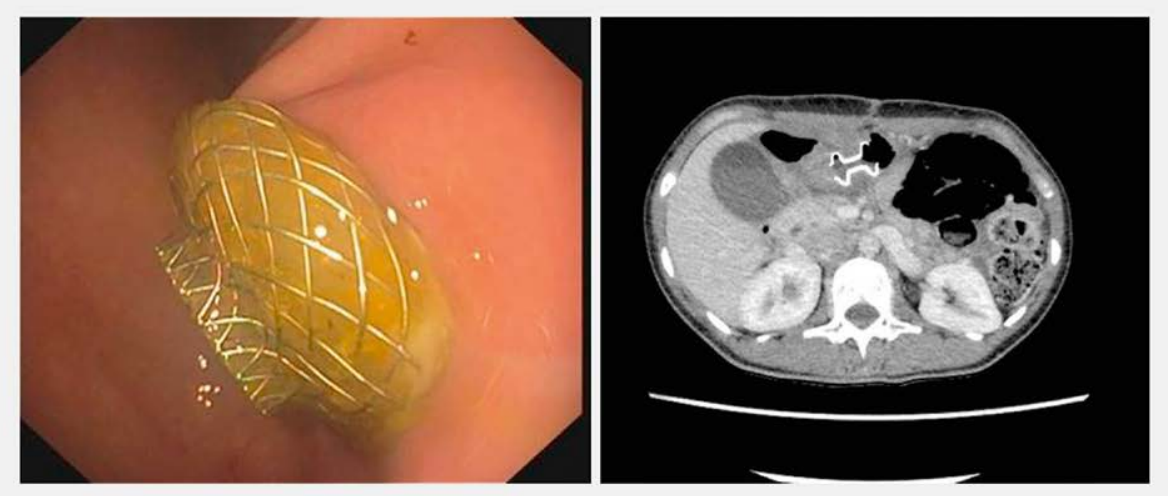

- Fig. 4 Gastroscopic view of the Hot Axios stent in the gastric lumen. CT scan with a Hot Axios stent between a collapsed pancreatic pseudocyst and the stomach. 


\section{Conflict of Interest}

The authors declare that they have no conflict of interest.

Authors

Hanne Soender Grossjohann', Thomas Skaarup Kristensen², Carsten Palnaes Hansen $^{1}$

Affiliations

1 Copenhagen University Hospital, Surgical Gastroenterology, Copenhagen, Denmark

2 Copenhagen University Hospital, Radiology, Copenhagen, Denmark
Bibliography

Dr. Hanne Soender Grossjohann, MD Copenhagen University Hospital,

Surgical Gastroenterology,

Blegdamsvej 9,

2100 Copenhagen,

Denmark

Tel.: +45/35/452122

hannesonder@dadlnet.dk
DOI https://doi.org/10.1055/a-0948-5620 Ultrasound Int Open 2019; 5: E75-E77 (c) Georg Thieme Verlag KG Stuttgart · New York

ISSN 2199-7152

(ㄷ) (1) (우) $\Theta$ 